\title{
EL CHACO BOLIVIANO: DEL PALEOINDIO AL PERÍODO ALFARERO TARDÍO
}

\author{
The Bolivian Chaco: From Paleoindian to the Late Ceramic Period
}

\section{Jorge A. Arellano*}

\section{Resumen}

El Chaco de Bolivia, que forma parte del Gran Chaco Sudamericano, tuvo en el pasado prehispánico un rol importante en las tierras bajas por su carácter de frontera natural entre dos ecosistemas y cuencas importantes: amazónica y chaqueña. A pesar de la información etnohistórica y etnográfica, esta zona marginal fue relegada en las investigaciones arqueológicas. En este artículo, integrando datos paleoambientales y arqueológicos, se presenta un nuevo análisis de la evolución del proceso cultural en el Chaco marginal, desde el paleoindio hasta el período alfarero tardío. En este sentido, se sugiere que cada evento ambiental importante está traducido en la adopción de diferentes sistemas y modos de vida en las poblaciones prehispánicas.

$<$ Bolivia Chaco Marginal $><$ Paleoambiente $><$ Paleoindio $><$ Período Alfarero $>$

\begin{abstract}
The Bolivian Chaco, northern marginal zone of the Great South American Chaco, had an important role during the pre-Hispanic periods because of its character of natural boundary between two main ecosystems and basins: Amazon and Chaco. Despite the ethnohistorical and ethnographic data, this marginal zone was relegated from the archaeological research. In this article, paleoenvironmental and archaeological data are integrated to analyze the process of cultural evolution in the Bolivian Chaco, from the paleoindian period to the late ceramic period. It is suggested that every major paleoenviromental event was reflected in the adoption of different systems and ways of life by the pre-Hispanic populations.
\end{abstract}

$<$ Bolivian Marginal Chaco $><$ Paleoenviroment $><$ Paleoindian $><$ Ceramic Period $>$

Recibido: 03/04/2014 // Aceptado: 15/07/2014

\footnotetext{
* Investigador Asociado del Departamento de Antropología del Museo de Historia Natural de la Smithsonian Institution - Whashington - USA, arellanoa@si.edu
} 


\section{Introducción}

El Chaco Boliviano forma parte de la región septentrional marginal del Gran Chaco Sudamericano y circunscribe el Chaco central semiárido y seco del Paraguay (Figura 1). De manera general esta región puede ser diferenciada en dos medioambientes: Chaco semi-húmedo, asociado a los cursos de los ríos Grande y Parapetí que forman los bañados del Izozog y Chaco seco que se extiende en la planicie aluvial al este del río Pilcomayo y al sur de la serranías de Sunsas y Chiquitos. En la frontera con Brasil, una limitada área del Chaco húmedo se desarrolla en asociación con los bañados de Tucavaca y el río Paraguay. Al mismo tiempo, en el período prehispánico tardío dos características culturales resaltan en estos particulares hábitats: la primera, que el Chaco semi-húmedo fue ocupado usualmente por grupos étnicos dedicados a la agricultura y la segunda, que el Chaco seco fue utilizado por varios grupos de cazadores recolectores.

\section{Figura 1. Chaco Marginal de Bolivia y principales sitios arqueológicos}

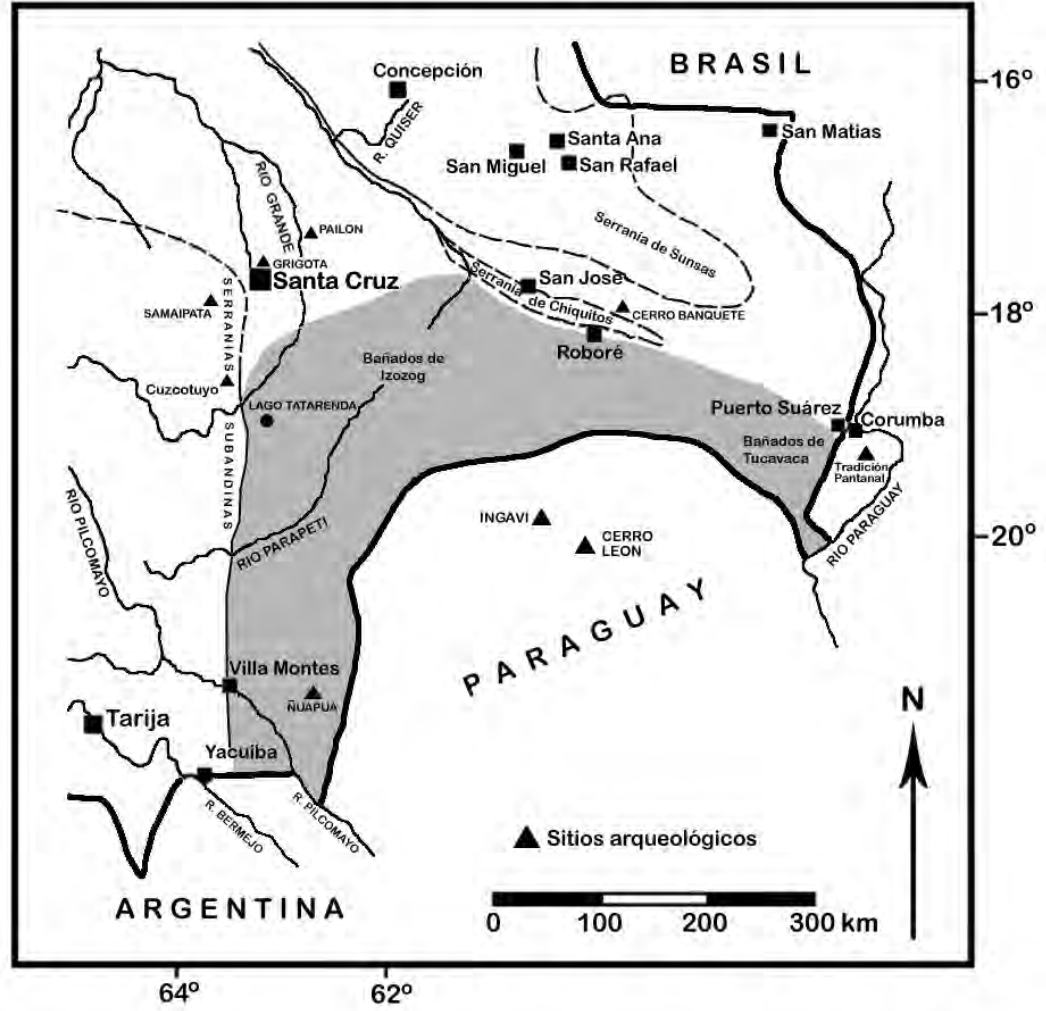

Fuente: Elaboración propia

Al respecto, es importante mencionar que las primeras descripciones de territorios culturales (Kersten, 1905) y mapas etnográficos, muestran que en el Chaco septentrional estuvieron asentados una diversidad de poblaciones étnicas. El mapa elaborado por Chervin (1908) indica la presencia de poblaciones chiriguanas al pie de 
las serranías subandinas, por el margen derecho del curso del Pilcomayo se encuentran los Chane, Piquirenda, Orales y Malbales, mientras en el margen izquierdo los Toba, Choroti y Taipieti. El mapa de De Nino (1912) indica a la derecha del Pilcomayo a los Matacos y Guisnais y a la izquierda los Tobas, Chorotis y Tapietis. Ambos coínciden en la falta de evidencias para el Chaco boreal. Por su parte, Metraux (1946) presenta un mapa con la localización geográfica de las tribus durante los primeros contactos con los europeos. Esta última versión tiene relevancia para la integración de datos arqueológicos con poblaciones prehispánicas y sus territorios de dominio. Desde el punto de vista de la cultura material de estas poblaciones históricas, los datos obtenidos por la expedición sueca Chaco-Cordillera (Von Rosen, 1924 a y b), son muy importantes por el material gráfico y descriptivo del patrón de asentamiento que pueden servir como punto de referencia para la interpretación del modo de vida en época prehispánica. Sin embargo, es muy difícil realizar una retrospectiva del pasado prehispánico de cada una de estas poblaciones, a esto se debe agregar la extinción de muchas con la desaparición de sus idiomas, tradiciones y cultura material.

Por el momento las pocas investigaciones arqueológicas centradas en el Chaco de Bolivia, Arellano (1986), Esquerdo (1998), Dames y More (2001), limitan la reconstrucción del desarrollo cultural de esta región con relación a los procesos culturales producidos en los Andes y las tierras bajas al norte de las serranías de San José y Sunsas. En este sentido, adquieren importancia las zonas periféricas al Chaco que tienen registros de las culturas prehispánicas que pudieron haber estado en contacto o utilizado la región marginal del Chaco como fuente alternativa de recursos naturales. Este último aspecto permite en cierta manera realizar algunas inferencias sobre el manejo prehispánico de su medioambiente y la ocupación de específicas áreas geográficas.

En consecuencia, el presente artículo tiene como objetivo actualizar la interpretación del proceso de desarrollo cultural en el Chaco Boliviano, en base a los nuevos datos del paleomedioambiente y las evidencias obtenidas en los sitios arqueológicos.

\section{Historia medioambiental del cuaternario reciente}

La historia geológica del cuaternario reciente del Chaco Boliviano en general es muy restringida. Las mejores inferencias para el Chaco de Bolivia pueden ser adaptadas de los estudios efectuados en la zona de las cuencas tarijeñas (Macfaden, 1981; Macfaden y Wolff, 1981; Isawaki y Arozqueta, 1982; Arozqueta y Lema, 1984) y en la pequeña cuenca de Nuapua, situada hacia el oriente de la serranía de Amarague que constituye el límite occidental del Chaco (Coltorti et al., 210; 2012), y de las investigaciones realizadas a lo largo del piedemonte andino marginal al Chaco Boliviano (May, 2006; May et al., 2008; Carnes, 2011). Los detallados perfiles estratigráficos de los depósitos sedimentarios de las cuencas de Tarija, muestran que durante el pleistoceno existió un lento proceso de cambio en las condiciones climáticas, con una continua tendencia al incremento de aridez, que como resultado produjo un paulatino desecamiento de los lagos para convertirse en ciénagas (Iwasaki y Arozqueta, 1982: 47) con la consiguiente extinción de megafauna. Mientras en Ñuapua, las evidencias indican que durante la 
transición pleistoceno-holoceno en el Chaco todavía existían cuencas con pequeñas lagunas de aguas estancadas, donde se reunían varias especies de megafauna extinta y otras especies pequeñas de anfibios y aves que sobrevivieron hasta la actualidad.

De manera particular los estudios efectuados por May (2006) y May et al. (2008) de la secuencia de paleosuelos en los cortes estratigráficos en las localidades de Cabezas y Pelícano, orillas del río Grande, límites marginales del Chaco, permiten inferir los eventos medioambientales que tuvieron impacto en el Chaco. En estas dos áreas se reconocieron 5 unidades (I, II, III, IV, V) de deposición de sedimentos que marcan diferentes períodos desde los efectos de la última máxima glaciación $(\geq 18000 \mathrm{cal}$ AP) al presente. Las unidades que podrían ser correlacionadas con Ñuapua son las tres superiores. Según May et al. (2008: 48), la unidad III en el período de transición entre pleistoceno y holoceno temprano, alrededor de cal. 11500 AP - 10000 AP, sugiere que el piedemonte andino anexo al Chaco marginal estuvo sujeto a extensas inundaciones por las altas precipitaciones invernales al este de los Andes. Por el contrario, en el Chaco existió un sistema fluvial estable que dio lugar a la deposición de sedimentos finos con la generalización de paleosuelos. Estas características indican que el Chaco marginal estuvo cubierto por una vegetación típica de llanura aluvial con áreas de lagunas pantanosas, tal como se menciona para sectores asociados al piedemonte cercano al río Grande (May et al. 2008: 48).

La unidad IV ubicada entre el holoceno temprano y medio, cal. 10000 AP $2900 \mathrm{AP}$, muestra que los eventos paleoambientales a partir de cal. $8000 \mathrm{AP}-7500 \mathrm{AP}$, estuvieron prácticamente asociados a una sedimentación eólica en las regiones aledañas a los ríos Pilcomayo y Parapetí (May et al., 2008: 49). La acumulación de sedimentos eólicos pudo haber continuado con dirección al Chaco central por los paleovientos provenientes del N-NW (May, 2006). Además es probable que los varios períodos de aridez dieron lugar a la propagación de incendios naturales en los bosques de Chiquitos, como los registrados entre el cal. 7800 AP y 5800 AP en el área de Santa Cruz (May et al., 2008: 49). Estos eventos también pueden ser correlacionados con las variaciones hidrológicas ocurridas en la laguna Opabusu conocida también como Tatarenda. La secuencia sedimentológica de esta laguna que se inicia en el cal. 5587 AP muestra que hacia el 1400 AP existió un importante evento de aridez (Carnes, 2011: 11). También los eventos de aridez ocurridos durante la transición del holoceno temprano al medio propiciaron la completa destrucción de los refugios forestales como el de Ñuapúa. Hacia los 3000 AP se inicia la formación de suelos estables paralelamente a una disminución de la actividad eólica y, en la cordillera oriental y el subandino el principio de las actuales condiciones climáticas. Por su parte, el Chaco marginal estuvo sujeto a una aridez inducida por la escasa precipitación de verano (May et al. 2008: 49).

La unidad $\mathrm{V}$ que se encuentra en la parte superior de los perfiles estratigráficos representa al holoceno tardío, muestran que a partir de cal. 2900 AP regresó la estabilidad geomorfológica con una expansión de bosques y hacia al presente el clima desarrolló fluctuaciones, especialmente extremas durante la presencia de los meganiños. Los registros que provienen de la cuenca de Cochabamba (Williams et al., 2011), sugieren 
para las tierras altas de la cordillera oriental y cabeceras de los valles centrales una alternancia de períodos húmedos y semi-húmedos (Williams et al., 2011). En tanto que en los llanos centrales existe formación e incremento de meandros en los ríos y en las tierras bajas del centro-sur, Chiquitos y Chaco, se presenta una actividad eólica con formación de dunas.

Estos datos sugieren que las condiciones medioambientales durante el holoceno medio y tardío en la cubierta de bosque amazónico por el norte y la llanura al sur de ella fueron prácticamente semejantes al actual. También existieron algunos períodos muy cortos de aridez usualmente producidos por los efectos colaterales del Niño y la Niña, y períodos de extrema humedad producidos por las precipitaciones del monzón de verano en Sudamérica (May et al. 2008: 36).

Como se puede observar, los eventos paleoambientales en las tierras bajas de Bolivia tuvieron diferencias en intensidad durante el cuaternario (Figura 2). Estos fueron mucho más acentuados en el pleistoceno puesto que siempre estuvieron a la expectativa de las ocurrencias de transgresión y regresión glacial en las montañas andinas.

Al inicio del holoceno estas diferencias establecieron una variedad de medioambientes con asociaciones vegetales específicas para cada región. Las corrientes fluviales de las tierras bajas de Chiquitos y el Chaco que comparten la misma cuenca sedimentaria con el río Amazonas, y que actualmente tienen dirección norte, probablemente tuvieron otro diseño en una diferente dirección. La mayor parte de los paleocanales y paleosuelos de la parte central de las tierras bajas no tiene concordancia con el diseño de drenaje actual, como demuestran los datos obtenidos en el estudio de tres mega-abanicos pluviales del piedemonte oriental: Piraí, Río Grande y Parapetí (May, 2006). Los paleocanales del río Piraí implican un cambio de dirección de su curso hacia el norte, y uno de sus paleocanales pudo ser mapeado hasta Paraguay indicando que fue parte de la cuenca del Río de la Plata (May, 2006: 126). Tal vez esta antigua superficie del paisaje a finales del pleistoceno y principio del holoceno determinó la diferente actitud y posterior adaptación de la cubierta vegetal. Indudablemente este antiguo paisaje afectó de igual manera a la forma de distribución de las primeras ocupaciones humanas.

\section{Paleoindio - arcaico}

En las cuencas de Tarija y Padcaya que contienen sedimentos del pleistoceno medio al tardío con especies extintas de megafauna no se encontraron evidencias asociadas a una presencia humana. Mientras en la pequeña cuenca de Nuapua que también contiene especies extintas, existe la probabilidad de una interacción humana-megafauna. El depósito cuaternario de Nuapua que se encuentra bastante erosionado tiene un espesor variable entre $7.10 \mathrm{~m}$ a $10 \mathrm{~m}$ y esta conformado por 3 unidades litológicas; la inferior Ñuapua 1, de origen fluvial compuesta por gravas y arenas fosilíferas; Nuapua 2, de origen lacustre con limos arcillosos y arcillas de color gris negruzco fosilíferas; Nuapua 3, limos arcillosos y suelo actual (Macfaden, 1981: 776; Coltorti et al. 2010; 2012). El área arqueológica está asociado a los sedimentos superiores de la unidad Nuapua 1 
que son parte de las orillas de una paleo-laguna y a los sedimentos de paleo-laguna que forman parte de Ñuapua 2. Aquí, de las capas inferiores fueron recuperados un cráneo y restos de un esqueleto humano asociados a restos de megafauna (MacFadden, 1981: 9;

Figura 2. Cuadro cronológico de los principales eventos paleoambientales en los valles centrales y tierras bajas del sur de Bolivia en relación con el Chaco Argentino-Paraguayo y Llanos Brasileño-Argentino

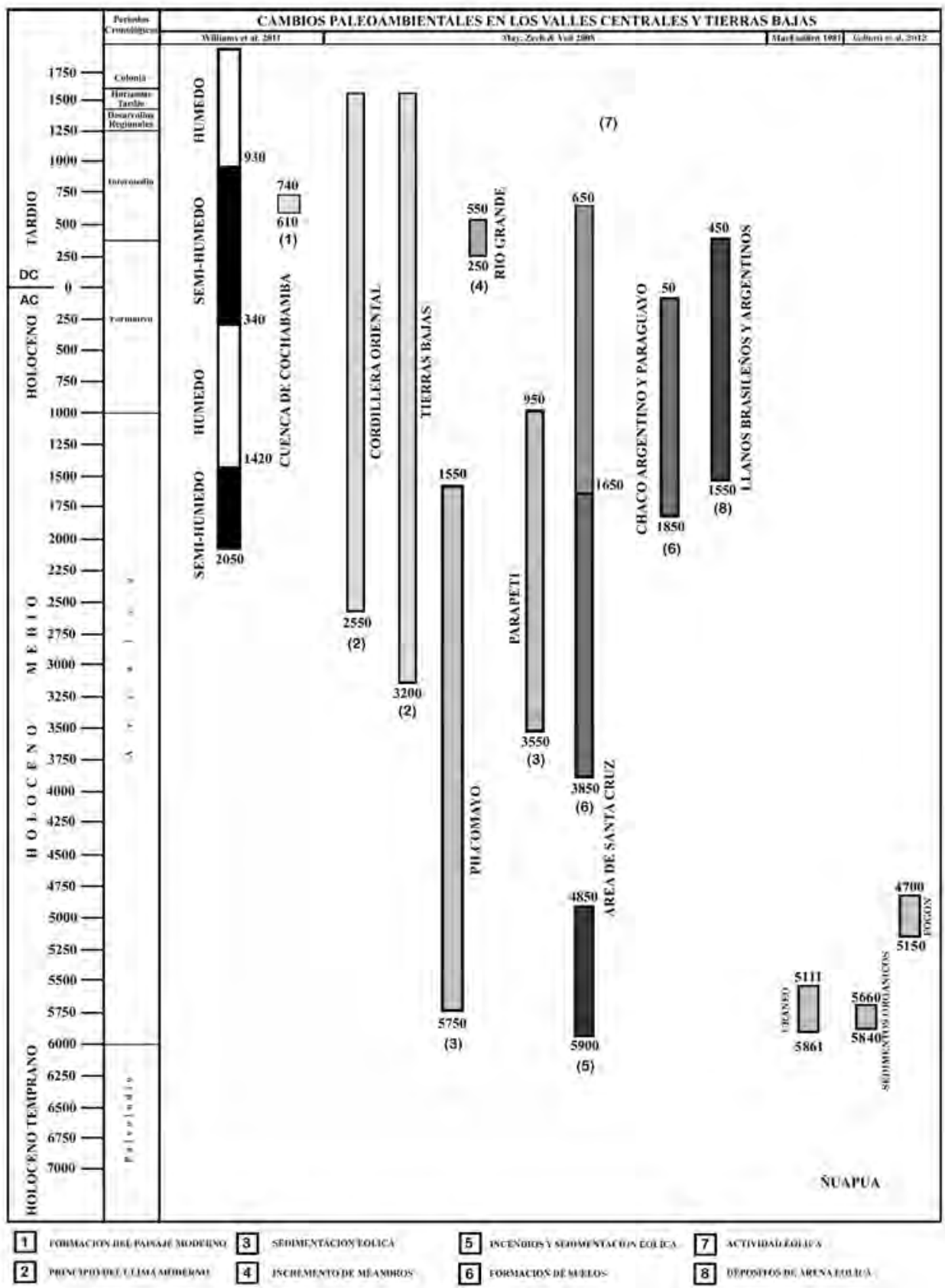

Fuente: Elaboración propia 
Macfaden y Wolff, 1981: 776). Análisis químicos han demostrado que el cráneo y los restos humanos (Figura 3) y megafauna, fueron cubiertos por sedimentos de la paleolaguna aproximadamente al mismo tiempo (Campbell, 1982: 30). El cráneo perteneció a un individuo del sexo femenino de 50 años de edad y fueron ubicados en asociación con restos de perezosos terrestres, caballos y paleolama (MacFadden, 1981: 9). No obstante, la datación por $\mathrm{C}^{14}$ del fósil humano sólo alcanzó a los $6600 \pm 370$ AP (cal. AP 7436 $\pm 375)$. Este aspecto en principio limita el espectro de su relación con la megafauna. Sin embargo las capas superiores de la unidad Ñuapua 1 también contienen artefactos líticos: Clastos y quijarros con tallas unifaciales y bifaciales, láminas, cuchillo-raedera, raspadores y perforador (Figura 4). Para la elaboración de los artefactos se utilizaron varios tipos de roca, cuarcita, cuarcita metamórfica, calcedonia (probablemente ftanita) y cuarzo (Arellano, 1986). La inexistencia de afloramientos rocosos en la zona indica que parte del material fue obtenido fuera de la región circundante a la paleo-laguna, mientras la cuarcita metamórfica pudo haber sido obtenida de los afloramientos de cuarcita gris de Cerro León, Chaco de Paraguay (Kuhn, 1991). Asimismo, el estrato que contiene los artefactos líticos presenta evidencias de fogones. Los fogones se encuentran dispuestos alrededor de las orillas de la paleo-laguna que estuvo en un proceso de desecamiento (Arellano, 1986:51), en correlación con los eventos medioambientales producidos en el piedemonte andino. Las especies de megafauna asociadas a los artefactos líticos y restos humanos pertenecen a Mylodontidae y Glyptodontidae (Arellano, 1986). Al margen de los restos de megafauna, en los sedimentos negruzcos de esta paleo-laguna se encuentran en mayor porcentaje restos fosilizados de aves y batracios (Arellano, 1986).

\section{Figura 3. Cráneo y restos de mandíbula del hombre prehistórico de Ñuapua}

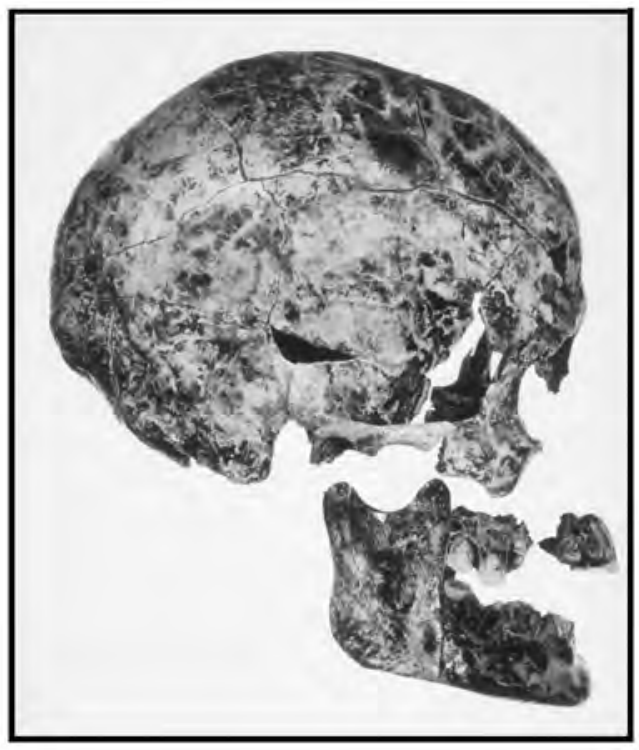

Fuente: Fotografía de B. MacFadden, 1982.

Resto depositado en el entonces Servicio Geológico de Bolivia, La Paz 


\section{Figura 4. Artefactos líticos de Ñuapua}

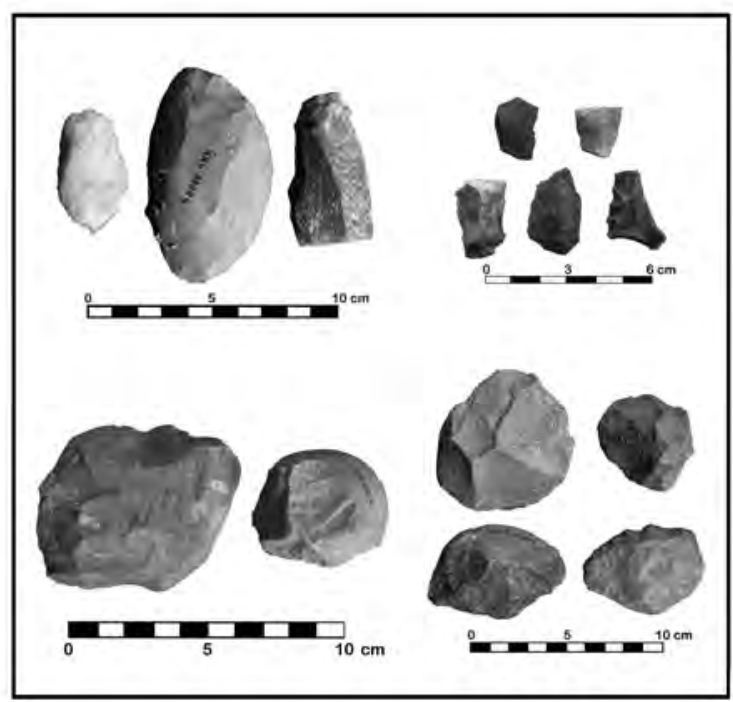

Fuente: Fotografía propia. Material depositado en el entonces Instituto Nacional de Arqueología, La Paz

Por encima de Ñuapua 2, se encuentra una delgada capa de ceniza volcánica o toba cuyo origen no fue posible determinar. La situación geográfica de Ñuapua con relación a la cadena volcánica de los Andes occidentales de Bolivia y la falta de estudios de los eventos volcánicos acaecidos durante el pleistoceno tardío y holoceno temprano, no permiten una correlación similar a las descritas en los Andes peruanos y ecuatorianos. De todas maneras, la toba volcánica marca un cambio medioambiental significativo puesto que las especies de megafauna fueron reducidas a una mínima variedad con relación al período anterior que está representado por el paquete de sedimentos del miembro Ñuapua 2 (MacFadden, 1981).

En la primera expedición a Ñuapua, MacFadden y Wolff (1981) también encontraron en el miembro superior Ñuapua 2, restos de Capromyidae y Toxodontidae y nuevas especies de Equidae, Camelidae y Cervidae. La presencia de estas nuevas especies y la variedad de anfibios y aves en el miembro Nuapua 2 indudablemente es producto del primer cambio medioambiental. Posteriormente la deposición de la capa de ceniza marca el segundo cambio, y con éste desaparecieron los Equidae y Camelidae.

Los últimos datos de Ñuapua proporcionados por Coltorti et al. $(2010,2012)$ corroboran lo mencionado en las investigaciones de McFadden (1981), Macfaden y Wolff (1981) y Arellano (1986). Las nuevas fechas radiocarbónicas provienen de la Unidad 1, Nuapua 1 de MacFadden (1981) y fueron obtenidas en restos de carbón de la parte superior de un fogón: $6870 \pm 50$ AP cal. 7790 a 7610 AP y del relleno de arena, limo y partículas de carbón de otro fogón: $5980 \pm 80$ AP cal. 7000 a 6650 AP (Coltorti et al. 2010: 221; 2012: 63). Estas fechas son prácticamente coincidentes con la obtenida 
por MacFadden (1981) en el resto óseo humano. De igual manera podrían confirmar la supervivencia de algunas especies de megafauna en refugios forestales por lo menos hasta 5500 AP.

La probabilidad de coexistencia humana y glyptodontidae también está registrada en el sitio La Moderna ubicado en la provincia de Buenos Aires, Argentina. En La Moderna se identificaron tres unidades litoestratigráficas dispuestas sobre sedimentos aluviales del Miembro Guerrero de la Formación Luján. La unidad inferior (ULa') está conformada por una transición de arenas limosas, limos arcillosos y arcillas limosas, y en la parte superior contienen "artefactos y desechos líticos asociados con restos óseos de Doedicurus clavicaudatus" (Politis y Gutierrez, 1998: 117). La unidad inmediatamente superior ULB en discordancia con la unidad ULa', contiene un limitado "conjunto lítico" de artefactos unifaciales y bifaciales asociados a restos de Lama guanicoe, Rhea americana, Canis cf. y Canis familiaris. La probable edad estimada para este contexto es "entre holoceno medio o inicios del holoceno tardío" (Politis y Gutierrez, 1998: 116-117).

Es bastante notoria la similitud de La Moderna con Nuapua en términos de la composición litológica, posición estratigráfica de las capas sedimentarias y relación con la presencia de una antigua laguna o pantano, características que sugiere eventos medioambientales correlacionables entre las regiones asociadas al Gran Chaco. De igual manera con el material lítico empleado en los artefactos, cuarzo, cuarcita y ftanita, y su descripción tipológica. La ubicación cronológica efectuada por $\mathrm{C}^{14}$ en los restos de Doedicurus clavicaudatus en asociación a los artefactos es estimada entre 7000 y 7500 AP (Politis y Gutierrez, 1998: 118).

Por su parte la tecnología de elaboración observada en el material lítico de Nuapua pudo haber sido transferida de los sitios paleoindio ubicados en el pantanal brasilero (Schmitz et al., 1998), a través de los sitios Ingavi y Cerro León situados en el Chaco Paraguayo (Alimen y Karpoff, 1967). El complejo lítico del sitio Ingavi asociado a una terraza del río Timane, está conformado por clastos con talla unifacial y bifacial, láminas unifaciales con retoque en los filos y láminas unifaciales con corteza original (Alimen y Karpoff, 1967: 871-75). Por su parte los artefactos de Cerro León son de mayor tamaño con talla unifacial y bifacial, y corteza residual (Alimen y Karpoff, 1967: 878-79). El tipo de roca empleado en los artefactos es cuarcita de color gris, probablemente de los depósitos aluvionales de grava conglomerádica originados por erosión de afloramientos de cuarcita de Cerro León. Para este complejo lítico no existe una posición cronológica definida y es considerado como precerámico (Alimen y Karpoff, 1967).

De manera que la evidencia más temprana disponible para inferir la ocupación humana en las tierras bajas del extremo sur de Bolivia es de 7000 AP, en refugios forestales con micro-medioambientes que contenían espacios lagunares favorables para la caza y recolección de especies vegetales. La composición de los sedimentos y espesor de las capas depositadas en la paleolaguna de Ñuapua, indican que estos pequeños oasis 
fueron inestables en la conservación del agua acumulada, y al mismo tiempo muestra una predominante escasez de precipitación pluvial.

\section{Período alfarero temprano}

Los pocos fragmentos de cerámica fueron ubicados en las capas superiores de la unidad Ñuapua 2, tienen rasgos de una tecnología formativa. Las características de la pasta y la decoración de esta cerámica, no tienen relación con las que muestran las corrientes de las tardías culturas Tupi-Guaraní. Las únicas formas reconstruidas son vasijas globulares abiertas tipo cuenco de "bordes directos y labios redondeados". Esta variedad de vasijas es común desde las etapas formativas, ya que son formas básicas que tienden a una fácil manufactura con materiales locales. Este pequeño complejo cerámico denominado Chaco Oriental, se caracteriza por su antiplástico de arena, paredes gruesas, con un acabado superficial alisado y pulido tosco (Figura 5). Se diferenciaron tres tipos, Pulido Tosco, Decorado Inciso y Decorado Punctuado (Arellano 1986: 54). Las decoraciones fueron efectuadas en fresco en el cuerpo de las vasijas y son bastante simples. El inciso está caracterizado por líneas finas semiparalelas alrededor del cuerpo y el punctuado fue efectuado en un ángulo, es profundo y dispuesto de manera desordenada en el cuerpo. A estos tipos se debe agregar el tipo Decorado Ungulado, impresión de uñas, observado en los fragmentos coleccionados por Coltorti et al. (2010: 225; 2012: 60 ), de un lente arenoso situado sobre un fogón del período paleoindio-arcaico, en un perfil estratigráfico erosionado. Este material obviamente proviene del estrato superior que contiene los fragmentos previamente descritos, los cuales por efecto de la erosión diferencial fueron depositados por gravedad sobre el fogón.

\section{Figura 5. Fragmentos de cerámica del período alfarero temprano de Ñuapua}

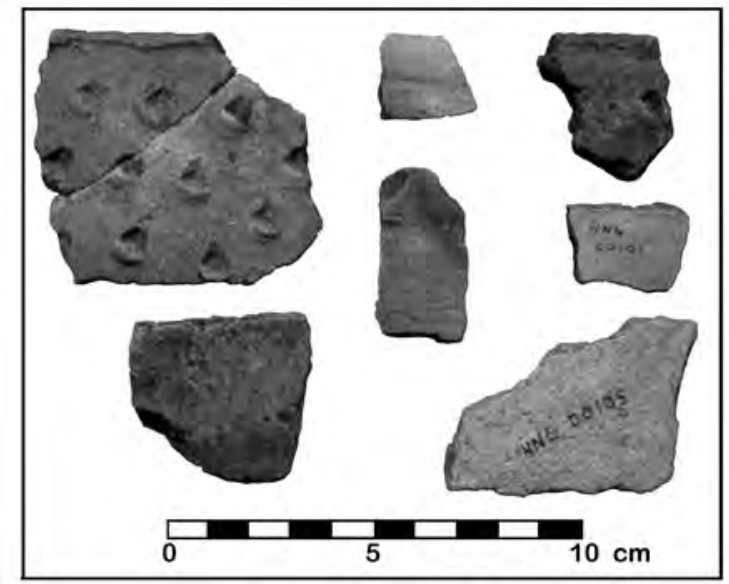

Fuente: Fotografía propia. Material depositado en el entonces Instituto Nacional de Arqueología, La Paz 
Estas variedades decorativas, punctuada y ungulada sobre superficies alisadas, en los sitios sobre el curso del río Ji-Paraná afluente del río Madeira, Rondonia, Amazonas de Brasil, se encuentran en un rango cronológico entre $3760 \pm 70$ AP a 2340 \pm 70 AP (Miller, 2009: 64,124).

La presencia de fragmentos de cerámica similares con decoración ungulada fue registrada en las excavaciones realizadas en la fortaleza Inka de Cuscotuyo ubicada en la frontera Inka del subandino. Este tipo denominado Parapetí Ungulado es considerado parte de la tradición Guaraní-Chiriguano que migró desde el este durante el denominado Ungulado Tardío-Inka entre 1480-1536 DC (Alconini, 2001: 413). Mientras en el Chaco central argentino al oeste del curso del río Pilcomayo, esta cerámica con decoración incisa y ungulada fue registrada en los sitios Pocitos, Pescado Negro y Encontrado, (Balbarrey et al., 2003). Aunque en estos sitios la cerámica predominante es la corrugada, es probable que los escasos datos de Nuapua indiquen desplazamientos tempranos de culturas no relacionadas con los Tupi-Guaraní y que Nuapua fue conocido como fuente de recursos para la práctica de recolección y caza hasta los períodos alfareros.

Por otra parte, las recientes investigaciones arqueológicas en el Chaco meridional argentino relacionadas con el sector ribereño del río Paraguay-Paraná mencionan que las primeras ocupaciones cronológicamente podrían ubicarse entre el 200 a 700 DC (Calandra y Salceda, 2006: 3). Según Lamenza et al. (2007), los sitios arqueológicos en el sistema ribereño Paraguay-Paraná se ubican en las orillas de cursos de agua secundarios, en un ambiente de vegetación densa y en terrenos altos. Esta característica es también muy particular en las ocupaciones prehispánicas que se encuentran en las orillas de los ríos amazónicos para evitar los flujos de las inundaciones en temporada de lluvias. La cerámica decorada con impresiones ungulares, y líneas incisas de los sitios en el Chaco central argentino tienen bastante similitud tecnológica con los presentes en el Chaco boliviano y sus fechas absolutas tienen un rango de 270 a 750 DC (Lamenza et al., 2007), y de acuerdo a Calandra y Salceda (2006: 10) "recién a partir del 500 DC comienzan a manifestarse la cerámica corrugada, corrugada incisa, pintura postcocción, cordelería impresa y filete aplicado".

\section{Período alfarero tardío}

En la franja marginal norte del Chaco (Figura 6), durante la implementación de la línea del gasoducto a Brasil, se realizaron reconocimientos arqueológicos en 12 áreas localizadas en las cercanías de corrientes fluviales, con estudios específicos en nueve sitios arqueológicos (Dames \& Moore, 2001: 49). En cada uno de los nueve sitios no se ha logrado diferenciar la sucesión de períodos de ocupación. El material cerámico fue analizado mediante la descripción de sus características físicas y decorativas, pero no se proporcionan los rangos verticales de la evolución de la cerámica con referencia a su localización en los depósitos culturales. Al parecer todos los sitios son multicomponentes y coexisten varias tradiciones cerámicas. 
Figura 6. Chaco marginal semi-húmedo. Mapa de ubicación de los sitios arqueológicos relacionados con el Río Grande y Bañados de Izozog

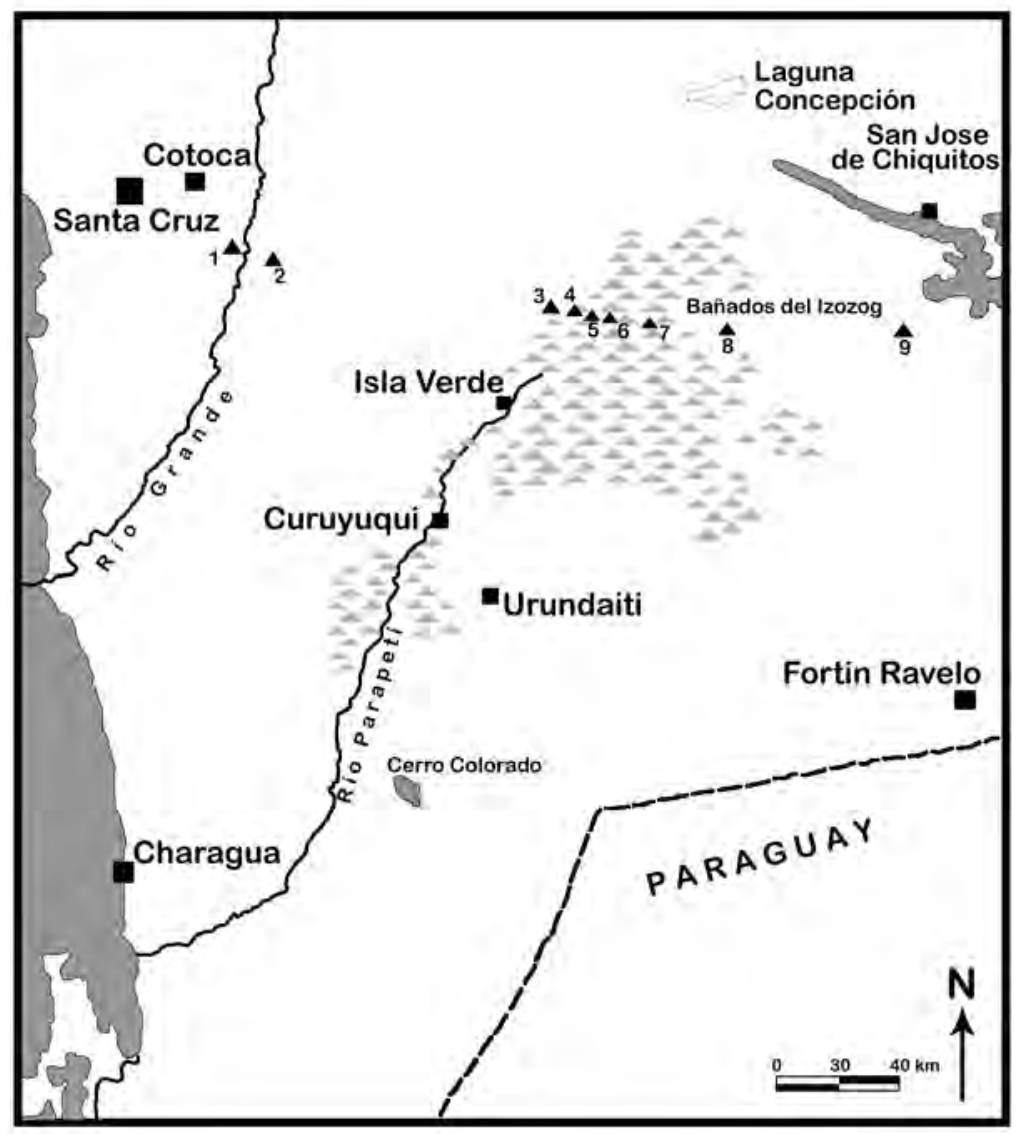

Fuente: Elaboración propia en base a los datos de Dames y More, 2001

El material cultural del sitio GBB-1, Río Grande, está conformado por fragmentos de los tipos alisado, escobado, corrugado, pintado e inciso. Las formas predominantes son vasijas globulares y cuencos trípodes de base plana. Adicionalmente se recuperaron "pesos de huso en forma de estrella, líticos no pulidos y una pequeña lámina de cobre". Por la presencia de cuencos trípode, este sitio fue relacionado con Pailon y Samaipata (Esquerdo, 1998: 84). Las dataciones $C^{14}$ en madera carbonizada muestran pocas diferencias en un rango entre 895 a 1119 DC. (Dames \& Moore, 2001: 77) sugiriendo que el sitio fue ocupado por un corto período.

El siguiente sitio denominado GBB-4, Parque Nacional Gran Chaco, está compuesto por varios montículos alrededor de una depresión interpretada como un reservorio (natural o artificial) de agua. En el material cerámico al margen del tipo alisado, está presente el tipo pintado negro sobre rojo que sugiere una relación andina, 
y el tipo inciso propio de las tierras bajas. La forma predominante son cuencos de los cuales el $80 \%$ corresponden a trípodes. Adicionalmente, se tienen tejos de cerámica, líticos y una lámina de cobre. Este último hallazgo también sugiere una relación con los Andes puesto que en el área no existen afloramientos de rocas con contenido de minerales de cobre.

Las dataciones del sitio GBB-4 se realizaron en muestras de tres contextos diferentes: con cerámica, con cerámica asociada a material óseo y de un fogón (Dames \& Moore, 2001: 186). Los resultados prácticamente son similares y el rango promedio es entre 1155 a 1268 DC. Este sitio es considerado como uno de los más extensos en el área inter-riverina y probablemente fue ocupado íntegramente en este período.

En el área de los Bañados del Izozog se ubicaron tres sitios, GBB-2, GBB-6, y GBB-7. El acabado de los fragmentos de cerámica recuperados indica que las vasijas fueron menos elaboradas. Entre las formas se presentan vasijas trípode con decoración ungulada y otros con decoración punteada e incisa (Esquerdo, 1998: 86). Una datación obtenida de madera carbonizada recuperada junto con dos vasijas y restos de plantas en el sitio GBB-6, proporcionó una fecha temprana de 770 a 965 DC (Dames \& Moore, 2001: 124). El análisis arqueo-botánico de los restos de plantas mostraron la presencia de Capsicum sp., ají y "dos diferentes tipos de fitolitos de Zea mays”. Sin embargo los análisis palinológicos no identificaron polen de Zea mays en los terrenos, indicando que el maíz no estuvo presente en el sitio (Dames \& Moore, 2001: 124). En el sitio GBB-7 se recuperaron dos entierros A y B. Los restos óseos del entierro A, fueron fechados en 833 a 837 DC, y los del entierro B, en 2860 a 2811 AC. Esta última fecha fue reconfirmada mediante otro análisis, además el rango cronológico de ocupación del sitio establecido por C-14 es de 1025 a 1205 DC (Dames y Moore, 2001: 132). Es interesante observar la variabilidad de las fechas, considerando que las obtenidas en los restos óseos se encuentran correctas significa que el sitio fue conocido desde el período Arcaico y que además fue producto de constantes reocupaciones.

Al sur de la población de Corumba en los límites con la República de Brasil (Figura 7), se ubica un conjunto de cuatro sitios en el área denominada Préstamo (Préstamo 1, 2, 3, y 4). El material cultural fue ubicado entre los 15 a $30 \mathrm{~cm}$ de profundidad. La presencia de idénticos tipos de cerámica, entre los cuales predomina el engobado en rojo sugiere que probablemente se trate de un solo grupo cultural con varias reocupaciones en el área. Los otros tipos de cerámica asociados son: Alisado, Inciso con motivos de líneas onduladas paralelas y triángulos, y Pintado rojo sobre anaranjado. En el sitio Préstamo-4 se presenta un tipo corrugado con bordes incisos. Las formas comunes son cuencos, ollas abiertas y urnas. El material complementario está compuesto por artefactos líticos (fragmentos de hachas), artefactos de cerámica (pesos de huso de forma cuadrangular y pipa fragmentada), y restos óseos de venado, Mazama sp. (Esquerdo, 1998: 40-56). En el sitio Calvario en la cima de una colina se recuperaron 20 fragmentos de cerámica de los tipos engobado en rojo, alisado e inciso con decoraciones en líneas paralelas. 
Figura 7. Chaco marginal húmedo. Mapa de ubicación de los sitios arqueológicos Préstamo, relacionados con la Cuenca del Río Paraguay

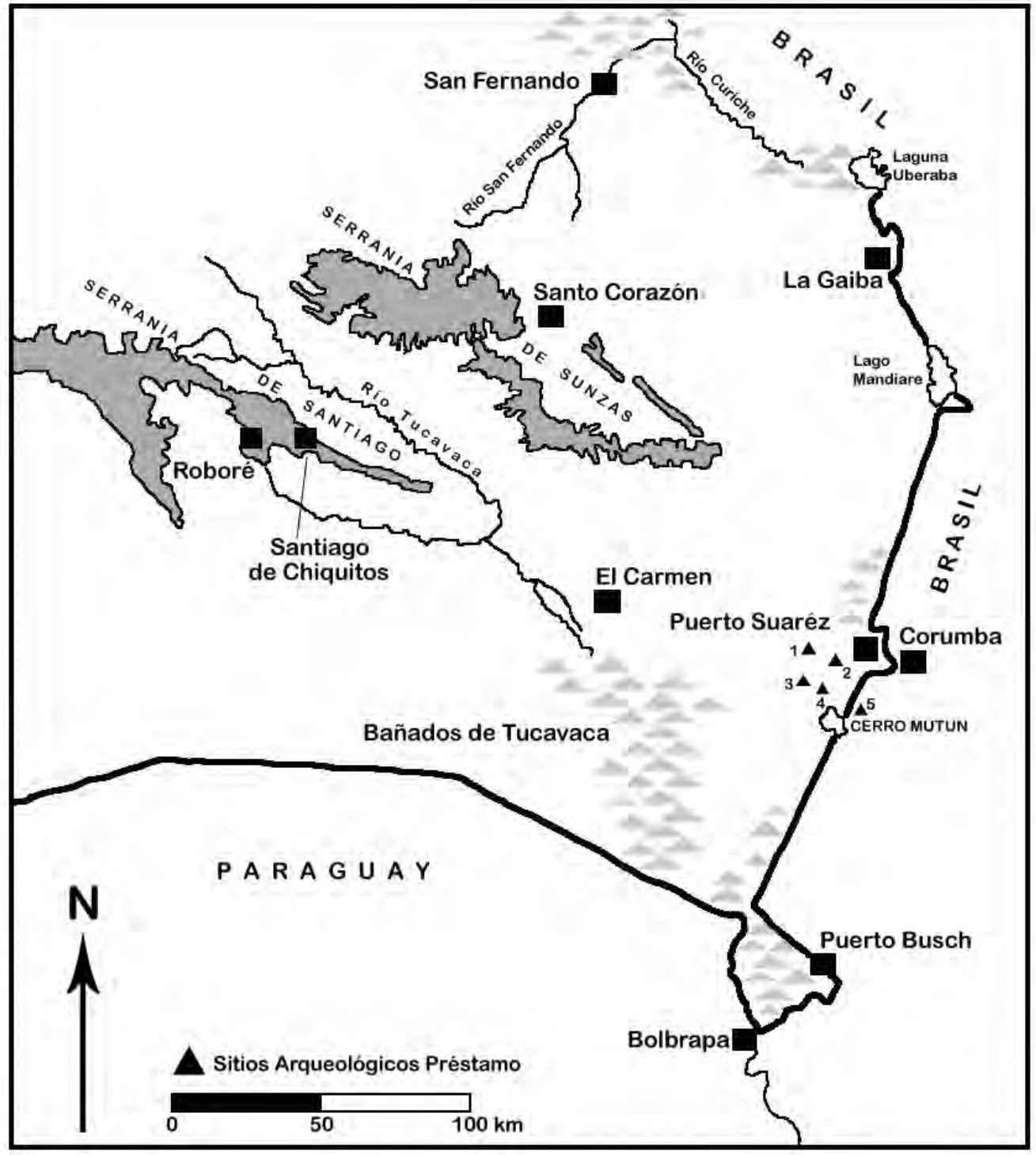

Fuente: Elaboración propia en base a los datos de W. Esquerdo Bernardo, 1998

Según Esquerdo, (1998: 92), los sitios Préstamo y Calvario, por la relación que tienen con el sistema fluvial Paraná-Paraguay-Uruguay, podrían pertenecer a una nueva fase de la sub-tradición guaraní con un rango cronológico entre los 1200 a 600 AP. No obstante, se puede inferir una relación temprana con la tradición Pantanal, Sur de la población de Corumba, Brasil, que tiene una variedad de estilos en la cerámica corrugada (corrugada simple, corrugada ungulada, corrugada digitada) cuya fecha más 
antigua es de 2160 AP (Schmitz et al., 1998: 223) y que parece extenderse hasta el Chaco paraguayo y argentino (Schmitz et al., 1998: 242). No obstante es importante hacer notar la presencia en Préstamo 2, de un fragmento decorado en rojo con motivos triangulares muy similares a la decoración incaica (Figura 8) y en Préstamo 4 la presencia de un fragmento de huso cuadrangular cercanamente parecido a los recuperados en Pailon 5, sitio en la orilla derecha del Río Grande, Norte de Santa Cruz, ocupado entre el 1040 a 1280 DC (Prümers y Winkler, 1997: 367, 383), y al recuperado en Chasqui en la zona del Valle de Ibirza en Chapare, ocupado en el 1449 DC (Brockington et al. 1995: 18). Esta variedad de huso está también presente en la fase Pantanal de Matto Grosso, en una fecha cercana al $1700 \pm 50$ DC (Schmitz et al. 1998: 225, 235). (Figura 9).

Figura 8. Fragmentos de cerámica del sitio Préstamo 4, se puede observar un probable fragmento de factura Inka

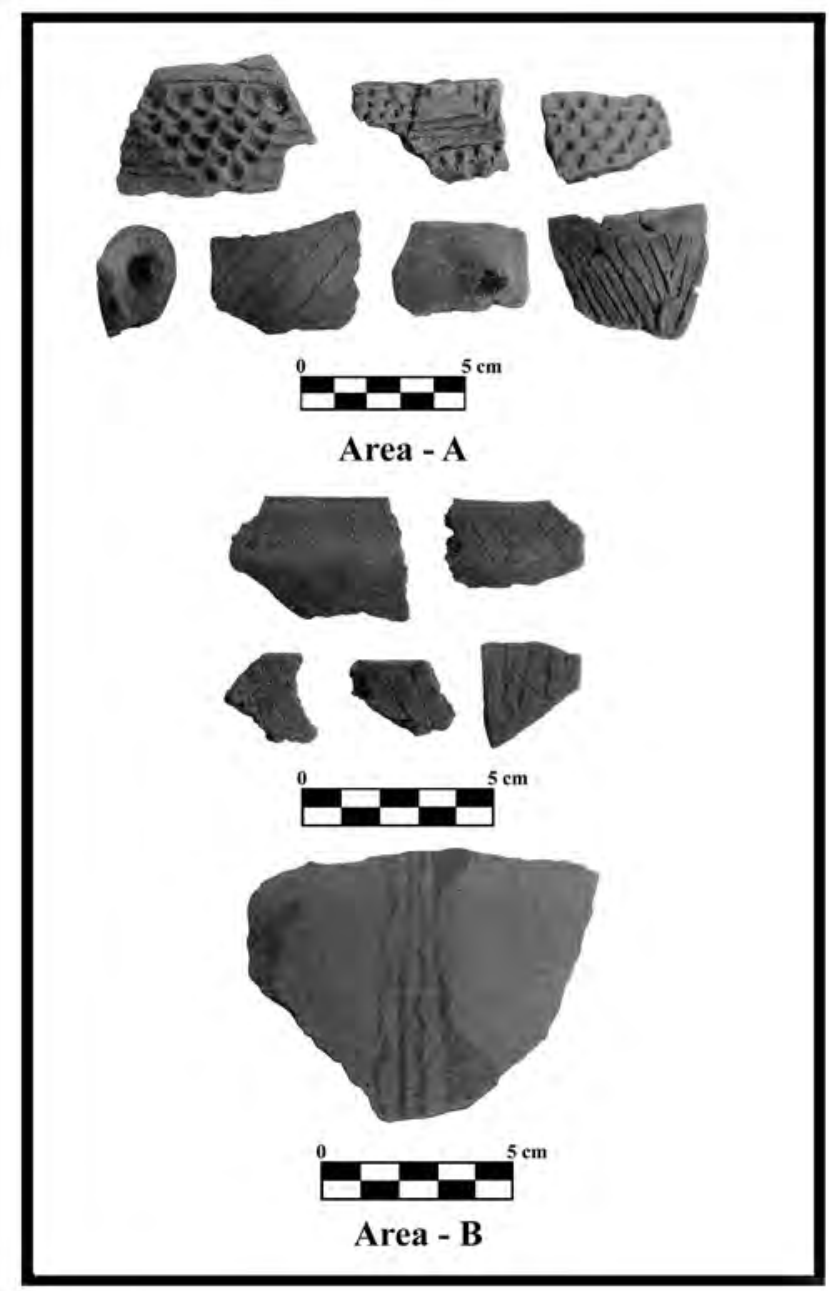

Funte: Elaboración propia en base a W. Esquerdo Bernardo, 1998 
Figura 9. Husos de cerámica probablemente empleados para hilar

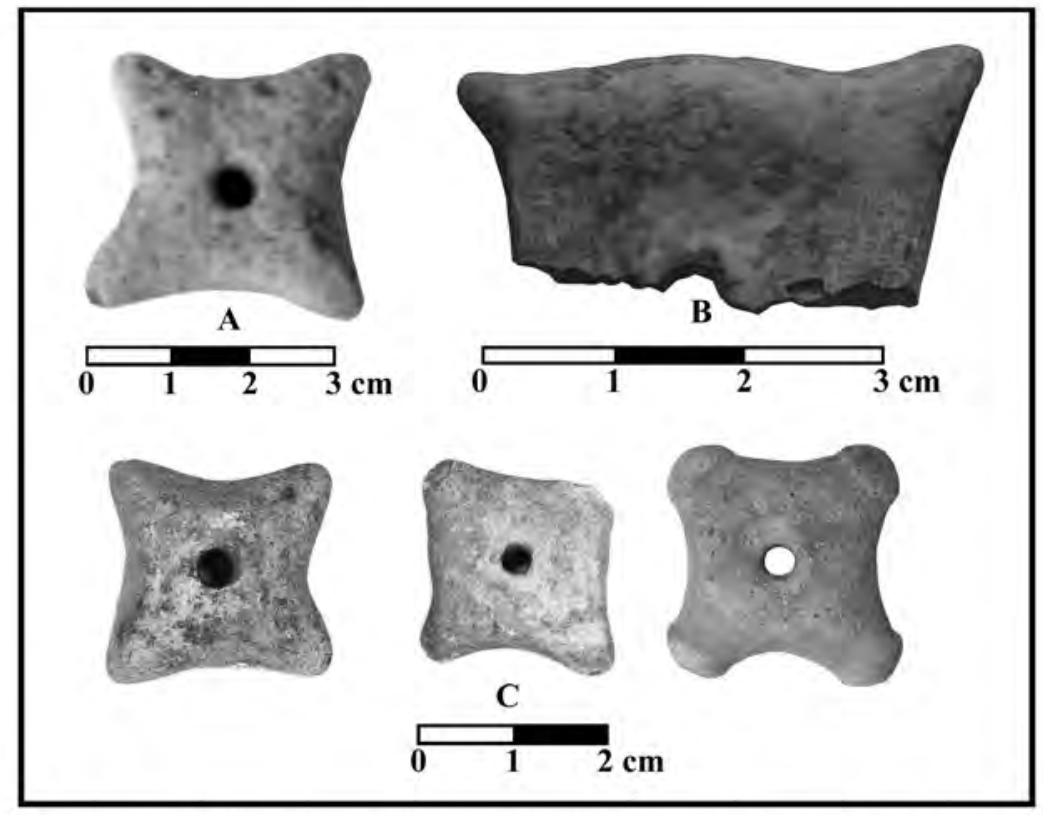

A: Chasqui, Villa Ibirza-Chapare B: Préstamo 4, Chaco-Sta. Cruz C: EI Pailon, Río Grande-Sta. Cruz

Fuente: Modificado de Brockington et al., 1995; Prümers y Winkler, 1997; Esquerdo, 1998

\section{Comentarios finales}

En el Chaco marginal los grupos culturales desde los períodos tempranos fueron bastante móviles y su limitada estabilidad tuvo relación con la renovación estacional de recursos naturales en los cursos de los ríos Pilcomayo y Parapetí y en oasis que formaron refugios forestales.

En consecuencia, el proceso histórico-social en el Chaco marginal probablemente se inició alrededor de los 7000 AP con la ocupación de refugios forestales, de los cuales con excepción de Ñuapua, todavía no se tienen registrados otros establecimientos paleoindio-arcaicos que permitan la correlación con los eventos del holoceno temprano. Los asentamientos paleoindios con fechas radiocarbónicas más cercanos se encuentran en el Brasil, en la serranía de Chapada dos Parecis y río Verde, donde afloran rocas del escudo brasileño que forman una serie de abrigos con evidencias de artefactos líticos agrupados en el denominado Complejo Dorado, situado cronológicamente entre el $8930 \pm 100$ AP cal. $9849 \pm 160$ AP y $10000 \pm 130$ AP; y $12473 \pm 214$ AP (Miller, 1987: 61). Sin embargo, el sitio MS-CP-22 situado en la zona del pantanal brasilero con una fecha radiocarbónica cal. $8160 \pm 60$ AP (Schmitz et al., 1998: 65), es importante porque presenta materia prima y material lítico afines a los de Nuapua. Sin embargo, el sitio La Moderna a pesar de encontrarse fuera de los límites del Gran Chaco adquiere 
importancia por la relación con megafauna y por proporcionar esta posibilidad en otros medioambientes.

En este sentido, tomando en cuenta que el período paleoindio comprende el rango cronológico que existe entre la transición del pleistoceno tardío al holoceno temprano (11000 a 10000 AP) y el arcaico con un rango entre 10000 a 6000 AP, podríamos considerar que las evidencias de Nuapua podrían sugerir la existencia de ocupaciones con características arcaicas en similares paleoambientes del Chaco como en los sitios Ingavi y Cerro León. En consecuencia Ñuapua se presentaría como una ocupación cronológicamente ubicada entre la transición del paleoindio al arcaico, aspecto que previamente fue analizado por Lynch (1990a: 146; 1990b: 16). De todas maneras, las condiciones medioambientales a partir de aproximadamente el 3000 AP fueron similares a las actuales y el Chaco se convirtió a decir de Metraux (1946: 210) en una zona transicional, ecológicamente y culturalmente.

El período alfarero temprano en el Chaco marginal probablemente tiene relación con la adopción de vasijas de cerámica por grupos arcaicos de cazadores recolectores. Si tomamos en cuenta que en la cerámica de los sitios tempranos del Chaco meridional, es usual la decoración con impresiones de uñas, punctuaciones e incisiones, es probable que los pocos fragmentos de cerámica del sitio de Ñuapua pertenezcan a un grupo temprano en proceso transhumante con dirección al subandino y otras áreas, o simplemente de cazadores recolectores itinerantes provenientes de las serranías de Chiquitos donde existen sitios cerámicos asociados a pinturas rupestres (Arellano et. al. 1977; Riester, 1981).

Esta última característica de inestabilidad y continua movilidad como tradición arraigada en poblaciones étnicas del Chaco, fue observada y descrita por D'Orbigny en 1831 en los grupos Guarañoca que habitan la zona Sur de la serranía de Chiquitos.

En el período alfarero tardío se observa que los asentamientos prehispánicos se ubican a lo largo del piedemonte andino en áreas cercanas a pequeñas corrientes fluviales, en el curso del Río Grande y en la planicie de los Bañados del Izozog. Las evidencias de los sitios ubicados en la transecta del gasoducto a Brasil, en un territorio previamente considerado culturalmente Tupi-Guaraní, indican que esta tradición no estuvo asentada en zonas sujetas a inundaciones estacionales. Las evidencias de vasijas de cerámica trípode hasta el área de los Bañados del Izozog sugieren un desplazamiento cultural a lo largo de los cursos de los ríos Piraí y Grande. Además, según Esquerdo (1998: 87), la presencia de una vasija antropomorfa en el sitio G-BB1 es una prueba que la tradición Tupi-Guaraní no ocupó los sitios mencionados.

Los datos etnohistóricos indican que los Chane fueron probablemente la etnia original asentada a los pies del subandino en los alrededores de los bañados del Izozog, y que esta fue la principal intermediaria entre las culturas Andinas y los pueblos de la planicie chaqueña con dirección hacia los bañados de Otuquis. Metraux (1946: 211) los menciona indicando que su rol fue difundir la cultura Andina y que posteriormente fueron esclavos de los Chiriguano, de los cuales adoptaron el idioma guaraní (Palavecino, 1949: 118). 
En relación a los Chiriguanos, según el mapa preliminar de asentamientos étnicos en el siglo XVI (Saignes s/f) y de Kersten (1905) del Gran Chaco, y los mapas de tribus indígenas en Bolivia de Chervin (1908), y de De Nino (1912), las etnias chiriguanas estuvieron formando una franja de ocupaciones desde el río Pirai por el norte hasta el río Bermejo por el sur. Las evidencias arqueológicas de restos de cerámica "chiriguana" ubicadas estratigráficamente por debajo y luego asociados a la cerámica Inka en Samaipata (Meyers, 2007), en las áreas de Ravelo, Monteagudo, el Ingre (Pärssinen y Siiriäinen, 2003) y Saipuru en el piedemonte del subandino (Combes, 2011), sugieren una convivencia natural de pueblos de frontera. En particular el sitio Saipuru es muy especial por la co-existencia de una diversidad de estilos cerámicos, además de las evidencias de objetos metálicos elaborados en plata, cobre y probablemente oro. La ubicación geográfica de Saipuru en una región fuera de la influencia Inka, sugiere que el sitio fue utilizado como mercado para intercambio de productos.

De manera que antes de la llegada de los Inkas las culturas del subandino estuvieron compartiendo estas áreas y sus recursos naturales. Lamentablemente, y por el momento no se tienen registrados sitios arqueológicos directamente relacionados con el piedemonte andino anexo al Chaco marginal, donde se encuentran asentadas la mayor parte de las etnías chiriguano.

Por su parte la cerámica de los sitios Prestamo del extremo marginal Este del Chaco boliviano, tienen relación con las tradiciones de la fase pantanal del Alto Paraguay (Schmitz et al. 1998), y con las fases culturales circunscritas en la confluencia de los ríos Paraguay-Paraná (Lamenza, 2013). De igual manera, tienen relación con el material cerámico del sitio Puerto 14 de Mayo, Bahía Negra, Paraguay (Lamenza, 2013: 130). En consecuencia es posible que los sitios asociados al sistema fluvial Pilcomayo, Paraguay, Paraná pertenezcan a una continua corriente de comunicación e intercambio entre varias fases culturales prehispánicas. Este mosaico de pueblos y etnías de las tierras del Chaco húmedo fue probablemente contactado por el primer europeo en las tierras bajas del Sur de Bolivia, el portugués Aleixo García, conquistador al servicio de España y miembro de la expedición de Juan Diaz de Solis, que conformó el pequeño ejército de nativos de varias etnías guaraníes asentadas en la orilla izquierda del río Paraguay, para internarse en el Chaco y llegar a los contrafuertes andinos (Nordenskiöld, 1917) hasta las tierras de los Chane y Caracara, Charcas (Metraux, 1946: 200). Estos grupos étnicos vendrían a ser los primeros grupos de guaraníes-chiriguanos que se enfrentaron a Huayna Kapac y que fueron tardíamente conocidos a través de los misioneros.

\section{Agradecimientos}

Deseo expresar mi agradecimiento al departamento de Antropología del Museo de Historia Natural de la Smithsonian Institution en la persona de la Dra. Mary Jo Arnoldi, Chair del Departamento, por darme la oportunidad de ser parte de esta prestigiosa Institución. Asimismo, al Dr. Dennis Stanford, Dr. Ronald Bishop y Dr. Paul Taylor, por su apoyo a la conclusión del presente trabajo de investigación. Mi agradecimiento al Dr. Kenneth Campbell del Museo de Historia Natural de Los Ángeles, por la oportunidad 
de realizar las investigaciones en el Chaco y Ñuapua. Finalmente, agradezco a los evaluadores anónimos por sus valiosos comentarios para mejorar el manuscrito.

\section{Referencias}

Alconini, S. 2004.” The Southern Inka frontier against the Chiriguanos: Structure and Dynamics of the Inka Imperial Borderlands". En: Latin American Antiquity 15 (4), pp. 389-418.

Alimen, H. y Karpoff, R. 1967. "Cailloutis quaternaries et pierres taillées du Chaco (Paraguay)". En: Bulletin de la Société Préhistorique Française. Études et travaux, T. 64 (3), pp. 865-884.

Arellano L., Jorge; Kuljis, D. y Kornfield, W. 1976. Pictografias del cerro Banquete (Sitio 8043031), Provincia Chiquitos, departamento de Santa Cruz. Instituto Nacional de Arqueología, Publicación No 17. La Paz, pp. 42.

Arellano, J. 1986. "Ñuapua, un asentamiento paleoindígena en Bolivia". En: Prehistoricas 1, Carrera de Antropología y Arqueología, UMSA, pp. 49-61.

Arozqueta, B. y Lema, A. 1984. "Nuevas Areas de Importancia en el Yacimiento Cuaternario de Tarija, Bolivia". En: On Fossil Mammals from the Tarija Department, Southern Bolivia. The Research Institute of Evolutionary Biology. Pub. No. 4, Tokio, pp. 54-58.

Balbarrey G.; Calandra, H.; Couso, G.; Lamenza, G. y Aguirre, M. B. 2003. Nuevos aportes al análisis cerámico del sector central del Gran Chaco Argentino. Facultad de Ciencias Naturales y Museo, UNLP, CONICET. (Ms. 18 pp.)

Brockington, D.; Pereira, D. y Sanzetenea, R. 1995. Archaeological Investigations at Chasqui, in the Eastern Lowlands of the Department of Cochabamba, Bolivia. (En Prensa), pp. 24.

Calandra, H. A. y Salceda, S. A. 2006. "Registro arqueológico regional chaqueño". En: Folia Histórica del Nordeste, No 16, Resistencia, Instituto de Investigaciones Geohistóricas-Conicet, Instituto de Historia-UNNE, pp. 1-13.

Campbell, K. E. 1982. "Bolivia: Cross Roads of a Continent". En: TERRA, Vol. 20, № 4. The Natural History Museum of Los Angeles, California, pp. 22-30.

Chervin, A. 1908. Anthropologie Bolivienne. Tome Premier; Ethnologie, Démographie. Mission Scientifique G. de Créqui Monfort et E. Sénéchal de la Grange. París, Imprimiere Nationale. pp. 407.

Carnes, A. 2011. Hydrologic variation and lake sediments: a reconstruction of the Bolivian Lowlands over the last 5500 years. Ms. Masters Project. The Nicholas School of the Environment, Duke University.

Coltorti, M.; Della Fazia, J.; Paredes, F. y Tito, G. 2010. "The Ñugapua alluvial fan sequence: Early and Late Holocene human-induced changes in the Bolivian Chaco?". En: Proceedings of the Geologists' Association 121, pp. 218-228.

Coltorti, M.; Della Fazia, J.; Paredes, F. y Tito, G. 2012. "Ñugapua (Chaco, Bolivia): Evidence for the latest occurrence of megafauna in association with human remains in South America". En: Journal of South American of Earth Sciences 33 , pp. 56-67. 
Combès, I. 2011. "Candire, Condori y Condorillo: presencia incaica en la cordillera chiriguana". En: Jean-Pierre Chaumeil, O. Espinosa y M. Cornejo (eds.) Por donde hay soplo. Estudios amazónicos en los países andinos. Instituto Francés de Estudios Andinos, PUCP, CAAAP, EREA. Lima, pp. 271-293.

Dames \& More Inc. 2001. East of the Andes, South of the Amazon. Archaeological Discoveries in the Dry Lowland forest of Bolivia. Imprenta Landivar, Santa Cruz, Bolivia. pp. 415.

De Nino, B. 1912. Etnografía Chiriguana. Imprenta Comercial de Ismael Argote, La Paz, Bolivia. pp. 334.

Diaz Arguedas, J. 1971. Expedicionarios y exploradores del suelo Boliviano. Tomos I y II, Ediciones Camarlingui. La Paz.

D’Orbigny, A. 1831 (1983). Viaje a la América Meridional. Tomo III. Editorial Futuro, Buenos Aires.

Esquerdo Bernardo, W. 1998. Evidencias de asentamientos humanos en la cuenca del Rio Paraguay (Provincia German Busch, Depto. De Santa Cruz) y su relación con el Gran Sistema Fluvial del Parana-Paraguay-Uruguay). Tesis de Grado, Carrera de Antropología y Arqueología, UMSA, La Paz, Bolivia.

Iwasaki, Y. y Arozqueta, B. 1982. "Geologic Background for the study of Mammal Paleontology of the Tarija, Bolivia". En: Tarija Bearing Formation in Bolivia. The Research Institute of Evolutionary Biology, Tokio, pp. 35-49.

Kersten, L. 1968 (1905). Las Tribus Indigenas del Gran Chaco Hasta Fines del Siglo XVIII. Una contribución a la Etnografía Histórica de Sudamérica. Universidad Nacional del Nordeste. Facultad de Humanidades, Departamento de Historia. Resistencia, Chaco, Argentina.

Kuhn, C. A. Clebsch. 1991. The Geological Evolution of the Paraguayan Chaco. Tesis de Doctorado, Texas Tech University, Texas, USA.

Lamenza, G.; Santini, M. y Castro, J. C. 2007. Ocupación del espacio en el sector ribereño Paraguay-Paraná (Chaco-Argentina). En: Segundo Encuentro de Discusión Arqueológica del Nordeste Argentino "Arqueología de cazadores recolectores en la Cuenca del Plata” Paraná, provincia Entre Ríos, República Argentina.

Lamenza, G. 2013. El Hombre y el Ambiente en el Holoceno Tardio del Chaco Meridional. UNCA, Universidad Nacional de Catamarca

Lynch, T. F., 1990a. "El hombre de la Edad Glacial en Suramérica: Una perspectiva Europea". En: Revista de Arqueología Americana, No 1, pp. 141-185.

Lynch, T. F., 1990b. "Glacial-Age Man in South America? A Critical Review". En: American Antiquity, Vol. 55 (1), pp. 12-36.

MacFaden, B. 1981. "Collecting fossils mammals in Bolivia. The southern connection". En: The Plaster Jacket. Florida Paleontological Society, Gainesville.

MacFaden, B. y Wolff, R. 1981. "Geological investigations of late Cenozoic vertebrate bearing deposits in southern Bolivia". En: Anais II Congresso Latino Americano de Paleontología, Porto Alegre, Brasil, pp. 765-778.

May, J. H. 2006. "Geomorphological indicators of large-scale climatic changes in the Eastern Bolivian lowlands". En: Geographica Helvetica 61 (2), pp. 120-134. 
May, J. H.; Zech, R. y Veit, H. 2008. "Late Quaternary paleosol-sediment-sequences and landscape evolution along the Andean piedmont, Bolivian Chaco". En: Geomorphology 98, pp. 34-54.

Metraux, A. 1946. "Etnography of the Chaco". En: Handbook of South American Indians. Vol. 1, The Marginal Tribes. Ed. Julian E. Steward, Smithsonian Institution, pp. 197-370.

Meyers, A. 2007."Toward a Reconceptualization of the Late Horizon and the Inka Period: Perspectives from Cochasquí, Ecuador, and Samaipata, Bolivia”. En: R. L. Burger, C. Morris, R. Matos, J. Pillsbury y J. Quilter (eds.) Variations in the Expression of Inka Power. Dumbarton Oaks Research Library and Collection, Washington DC, pp. 223-254.

Miller, E. Th. 1987. "Pesquisas arqueólogicas paleoindígenas no Brasil Occidental”. En: Estudios Atacameños 8, pp. 37-61.

Miller, E. Th. 2009. "A cultura cerâmica do Tronco Tupi no alto Ji-Parana, Rondônia, Brasil: Algumas Reflexões teóricas, Hipotéticas e Conclusivas”. En: Linguística Antropológica, Vol. 1, № 1, pp. 34-136.

Nordenskiöld, E. von. 1917. "The Guarani Invasion of the Inca Empire in the Sixteenth Century: An Historical Indian Migration”. En: Geographical Review, Vol. 4, No. 2, American Geographical Society, pp. 103-121.

Palavecino, E. 1949. “Algunas Informaciones de Introducción a un Estudio sobre los Chane”. En: Revista del Museo de La Plata (Nueva Serie), Tomo IV. Sección Antropología, pp. 117-131.

Pärssinen, M. y Siriänen, A. 2003. Andes Orientales y Amazonia Occidental. Producciones CIMA. La Paz, Bolivia.

Politis, G. G. y Gutiérrez, M. A. 1998. "Gliptodontes y Cazadores-recolectores de la Región Pampeana (Argentina)". En: Latin American Antiquity Vol. 9, N 2, pp.111-134.

Prümers, H. y Winkler, W. 1997. "Archaologische Untersuchungen im bolivianischen Tiefland, Erster Bericht des Projektes Grigota”. En: Sonderdruck aus Beitrage zur Allgemeinen und Vergleichenden Archaologie. Band 17, Mainz, pp. 343-393.

Prümers, H. 2000. Der Fundort Grigota in Santa Cruz de la Sierra (Bolivien). En: Sonderdruck aus Beitrage zur Allegemeinen und Vergleichenden Archaologie. Band 20, Mainz, pp. 205-259.

Riester, J. G. 1981. Arqueología y Arte Rupestre en el Oriente Boliviano. Editorial Amigos del Libro, La Paz, 232 pp.

Saignes, Th. s/f. Evidencias históricas para establecer un mapa étnico del Sur andino pre-Inca: Problemas Metodológicos. Manuscrito. 5 pp.

Schmitz, P. I.; Rogge, J. H. ; Osorio Rosa, A. y Beber, M. V. 1998. Aterros Indígenas no Pantanal do Matto Grosso do Sul. En: Pesquisas Antropología 54, Instituto Anachietano de Pesquisas, Río Grande do Sul, pp. 1-271.

Von Rosen, E. 1924a. Popular Account of archaeological research during the Swedish Chaco-Cordillera Expedition 1901-1902. C. E. Fritze Ltd. Stockholm, pp. 168. 
Von Rosen, E. 1924b. Ethographical research work during the Swedish ChacoCoridllera Expedition 1901-1902. C. E. Fritze Ltd. Stocklhom, pp. 284.

Williams, J. J.; Gosling, W. D.; Coe, A. L. y Brooks, S. J. 2011."Four thousand years of environmental change and human activity in the Cochabamba Basin, Bolivia". En: Quaternary Research, Vol. 76. (1), pp. 58-68. 